\title{
CLASSIFICATION OF VARIOUS LAND FEATURES USING RISAT-1 DUAL POLARIMETRIC DATA
}

\author{
V. N. Mishra, P. Kumar, D. K. Gupta, R. Prasad \\ Department of Physics, Indian Institute of Technology (BHU), Varanasi- (vnmishra.rs.app13, pradeep.rs.app, dkgupta.rs.app, \\ rprasad.app)@itbhu.ac.in
}

KEY WORDS: Land use land cover, Dual Polarimetric, RISAT-1, Transformed Divergence, SVM

\begin{abstract}
:
Land use land cover classification is one of the widely used applications in the field of remote sensing. Accurate land use land cover maps derived from remotely sensed data is a requirement for analyzing many socio-ecological concerns. The present study investigates the capabilities of dual polarimetric C-band SAR data for land use land cover classification. The MRS mode level 1 product of RISAT-1 with dual polarization (HH \& HV) covering a part of Varanasi district, Uttar Pradesh, India is analyzed for classifying various land features. In order to increase the amount of information in dual-polarized SAR data, a band $\mathrm{HH}+\mathrm{HV}$ is introduced to make use of the original two polarizations. Transformed Divergence (TD) procedure for class separability analysis is performed to evaluate the quality of the statistics prior to image classification. For most of the class pairs the TD values are greater than 1.9 which indicates that the classes have good separability. Non-parametric classifier Support Vector Machine (SVM) is used to classify RISAT-1 data with optimized polarization combination into five land use land cover classes like urban land, agricultural land, fallow land, vegetation and water bodies. The overall classification accuracy achieved by SVM is $95.23 \%$ with Kappa coefficient 0.9350 .
\end{abstract}

\section{INTRODUCTION}

The accurate and timely land use land cover (LULC) information is essential for analyzing many socio-ecological concerns. Remote sensing data obtained from various optical sensors have been frequently used to derive LULC information (Saatchi et al., 1997; Roberts et al., 2003; Thenkabail et al., 2005). However, the conventional optical remote sensing is inadequate because of weather conditions. So, the difficulties are encountered in collecting timely LULC information. Microwave remote sensing have the capability of penetrating through the clouds thus overcoming the atmospheric effects and is therefore an effective tool for extracting timely LULC information. Earlier the space shuttle SIR-C/X-SAR data has been mainly used to investigate LULC information (Saatchi et al., 1997; Pierce et al., 1998).

The Classification of SAR images has drawn a lot of attention after the availability of the polarimetric SAR images through ENVISAT ASAR, ALOS PALSAR, Radarsat-2, RISAT-1 and SIR-C etc. However, several factors such as the complexity of the landscape in a study area, type of remotely sensed data and classification methods may influence the success of the classification of remotely sensed data. Thus, it is preferable to choose more advance classifier to attain more accurate results for land used land cover classification. Several classification algorithms have been reported for classifying various satellite images (Townshend, 1992; Hall et al., 1995). In recent years, the use of Support vector machine classifier has been increased significantly for land use land cover classification of remotely sensed data

The conventional parametric classifiers, such as the maximum Likelihood (ML) classifier is widely used (Huang at al., 2002; Waske and Braun, 2009) because they provide acceptable accuracy in spite of its limitations due to normal distribution of class signature (Swain and Davis, 1978). Unlike parametric classifiers, the non-parametric classifiers such as Artificial Neural Network (ANN) or Support vector machine (SVM) is not influenced by the assumption of normal distribution of data

* Corresponding author
(Dixon and Candade, 2008; Foody, 2004) and therefore they are more appropriate for classifying remotely sensed data.

SVM, a recently developed classifier based on machine learning theory (Vapnik, 1999) provides some system-inherent advantages in comparison with other classification algorithms. It has been revealed to achieve higher accuracies in LULC mapping and surpass other classification algorithms (Huang et al., 2002; Foody and Mathur, 2004; Mountrakis et al., 2011). It is mostly applied to hyperspectral data however few studies are also conducted for SAR data classification (Mercier et al., 2000; Fukuda et al., 2001; Krishnapuram et al., 2003). The classification of SAR and optical datasets using SVM classifier as well as a method based on the fusion of SVMs surpassed all other parametric and nonparametric classification techniques with more than 3\% accuracy improvement (Waske and Benediktsson, 2007). Many researchers have been shown that SVMs are not relatively sensitive to training sample size and it can work even with limited quantity and quality of training data (Mountrakis et al., 2011). The class separability analysis is also performed using Transformed Divergence (TD) procedure which measures the separability between classes and therefore may be used to evaluate the quality of the statistics prior to image classification.

In the present paper, the potential of non-parametric SVM classifier is analyzed for classifying RISAT-1 dual polarimetric data covering a part of Varanasi district of Uttar Pradesh for the year of 2013. Further, the classification accuracy for various land features is also examined.

\section{STUDY AREA AND DATA USED}

A part of Varanasi district of Uttar Pradesh, located in northern part of India has been selected as the study area. It has centre latitude $25^{\circ} 17^{\prime} 51.19^{\prime \prime} \mathrm{N}$ and longitude 82 $56^{\prime} 36.74^{\prime \prime} \mathrm{E}$ and covers $446.97 \mathrm{Km}^{2}$ area. The Varanasi (also known as Kashi) district located on the bank of Holy River Ganga is one of the oldest living cities in the world. The land is very fertile and wealthy in agriculture due to its location at the Indo-Gangetic plain. 
Dual polarimetric medium resolution (MRS) mode of RISAT-1 data (C-band) with incident angle $36.85^{\circ}$ has been utilized for the present study. The Image is acquired on 9th August 2013. Simultaneous field visit is also conducted to collect ancillary ground truth information required for the classification and analysis. The location map of the study area with hybrid false colour composite (FCC) of the RISAT-1 data is shown in Figure 1.

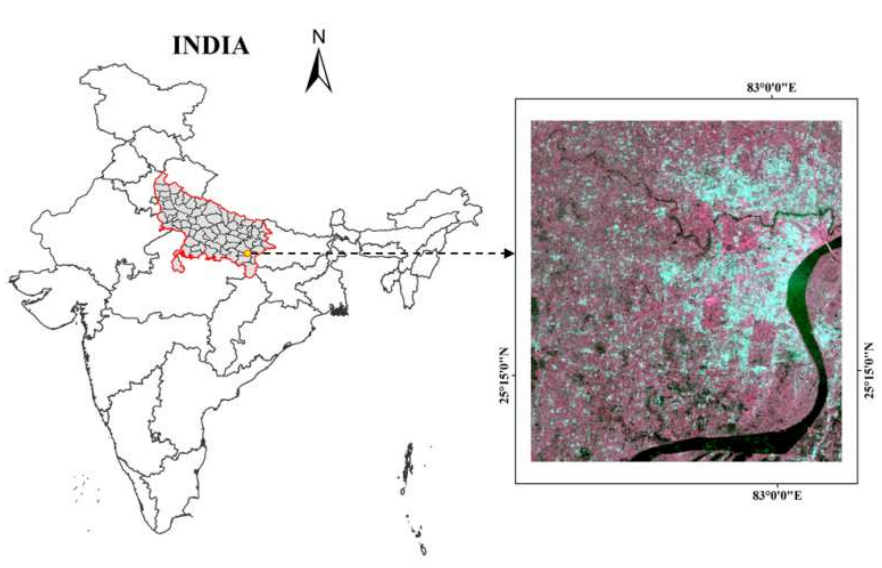

Figure 1. Location of the study area with RISAT-1 Hybrid FCC image (Red-HV, Green-HH, Blue-HH+HV)

\section{METHODOLOGY}

\subsection{Image pre-processing}

The level 1 (L1) product of RISAT-1 data in SLC (single look complex) format is first imported into SARscape using ENVI 5.1 software. It has $\mathrm{HH}, \mathrm{HV}$ polarization and 25 meter spatial resolution. The RISAT- 1 data is multilooked 2 times in azimuth and 2 times in range direction. Speckles appear as grainy "salt and pepper" texture in SAR imageries so it is required to reduce for interpretation and classification. The SAR data is filtered using Lee filter with $5 \times 5$ window size to remove the speckle noise. The Geocoding is performed by using leader file information of the data and the digital number (DN) values of C-band RISAT-1 data is converted into backscattering values in decibel $(\mathrm{dB})$ unit for $\mathrm{HH}$ and $\mathrm{HV}$ polarization. The amount of information in dual polarized data is increased by introducing a band $\mathrm{HH}+\mathrm{HV}$ based on the original two polarizations. The RISAT-1 data with three different polarizations $\mathrm{HH}, \mathrm{HV}$ and $\mathrm{HH}+\mathrm{HV}$ are layer stacked to get multipolarized image in order to acquire more clear discrimination between land features. The major steps of image classification include feature extraction, selecting training samples and finally classifying the data using suitable classification approach.

\subsection{Class separability analysis}

Training samples are defined for five major target classes such as urban area, agricultural land, fallow land, vegetation and water bodies. The class seperability analysis is performed using Transformed Divergence (TD) procedure between classes and may be used to assess separability of LULC classes in advance to image classification. It is a measure of statistical distance between classes and calculated from means and covariance matrices of each class. The values range from 0 to 2.0 and indicate how well the selected training samples are statistically separate. The values greater than 1.9 indicate that the classes have good separability. Transformed divergence can be calculated using the formula (Swain and Davis, 1978) as shown below,

$\mathrm{TD}_{\mathrm{ij}}=2\left(1-\exp \left(\frac{-\mathrm{D}_{\mathrm{ij}}}{8}\right)\right)$

Here, $\mathrm{Dij}=$ divergence between two signatures and can be calculated by

$$
\begin{aligned}
\mathrm{D}_{\mathrm{ij}}= & \frac{1}{2} \operatorname{tr}\left(\left(\mathrm{C}_{\mathrm{i}}-\mathrm{C}_{\mathrm{j}}\right)\left(\mathrm{C}_{\mathrm{i}}^{-1}-\mathrm{C}_{\mathrm{j}}^{-1}\right)\right) \\
& +\frac{1}{2} \operatorname{tr}\left(\left(\mathrm{C}_{\mathrm{i}}^{-1}-\mathrm{C}_{\mathrm{J}}^{-1}\right)\left(\mu_{\mathrm{i}}-\mu_{\mathrm{j}}\right)\left(\mu_{\mathrm{i}}-\mu_{\mathrm{j}}\right)^{\mathrm{T}}\right)
\end{aligned}
$$

Where,

$\mathrm{i}$ and $\mathrm{j}=$ the two signatures (classes) being compared

$\mathrm{C}_{\mathrm{i}}=$ the covariance matrix of signature $\mathrm{i}$

$\mu_{\mathrm{i}}=$ the mean vector of signature $\mathrm{i}$

$\operatorname{tr}=$ the trace function which calculates the sum of the elements on the main diagonal

$\mathrm{T}=$ the transpose of the matrix

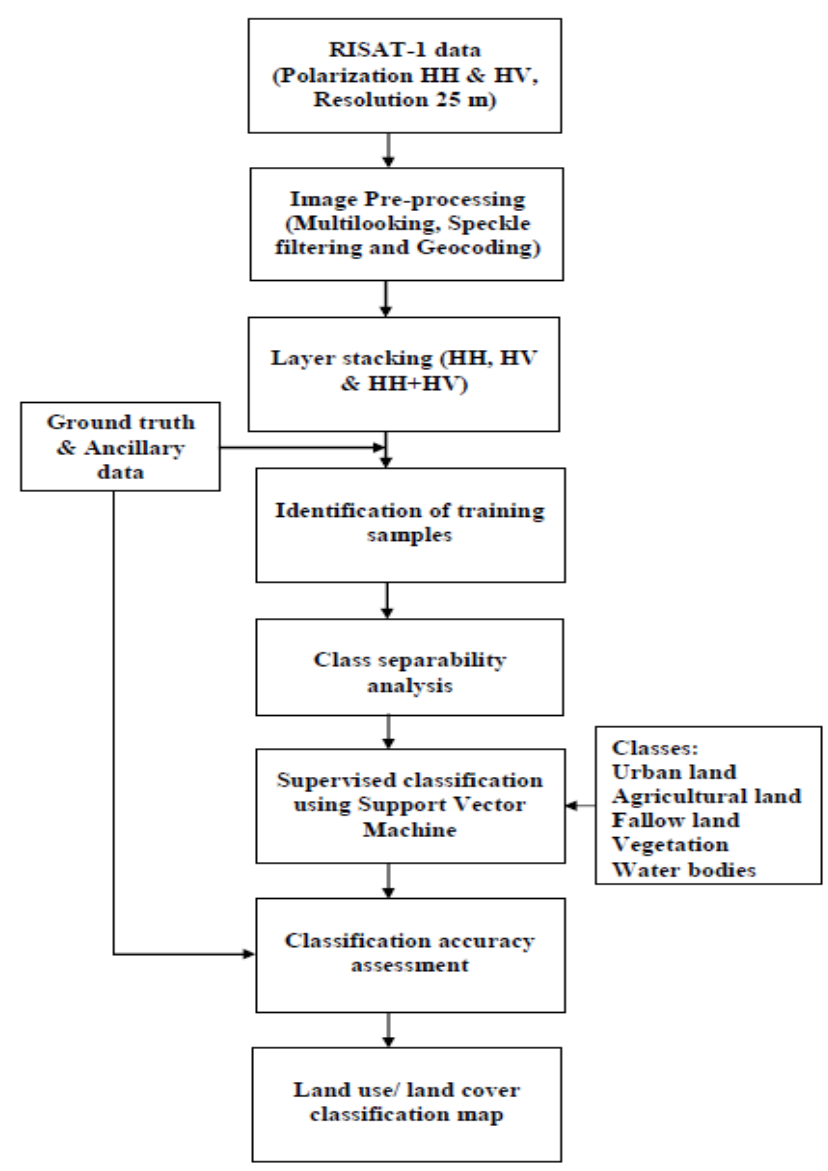

Figure 2. Methodology for the present study

\subsection{Support Vector Machine classification method}

The supervised classification has been carried out on SAR data with optimum polarization combination with the help of identified training samples. SVM is a recently developed statistical classifier based on machine learning theory. It uses structural risk minimization (SRM) principle to minimize the probability of misclassifying a previously unseen data point drawn randomly from a fixed but unknown probability distribution (Vapnik, 1995; Perkins et al., 2001). The aim of 
SVM is to separate two classes by fitting an optimal separating hyperplane to the training data. It provides the best separation between two classes within a multidimensional feature space using only the closest training data (Vapnik, 1998; Waske and Benediktsson, 2007). This hyperplane is a decision surface and is constructed by maximizing the margin between the class boundaries. The data points closest to the hyperplane are called "support vectors" (Cortes and Vapnik, 1995).

SVMs were primarily designed for binary (two-class) problems. A number of methods have been proposed to construct multiclass classifiers using two-class methods (Hsu and Lin, 2002). If two classes are not linearly separable, the concept of the kernel is proposed to handle the classification problems (Cortes and Vapnik, 1995). Radial basis function can be used for linear separation of class distributions with non-linear boundaries into a high dimensional space (Huang et al., 2002). In this study radial basis function (RBF) kernel method is chosen and it worked well in most of the conditions. The default value of penalty parameter and gamma parameter $(\gamma=0.33)$ are used in the present study. The training, classification, and accuracy assessment are carried out using ENVI 5.1 software. The proposed methodology is shown in Figure 2.

\subsection{Classification accuracy assessment}

The accuracy of SVM based classification result is assessed by computing the overall accuracy, user's accuracy, producer's accuracy, and Kappa statistics (Congalton and Green, 1999). The overall accuracy of classification result is evaluated by using the contingency matrix approach. The contingency matrix compares the correlation between well known reference data and the corresponding automated classification results on category by category basis. The overall accuracy is calculated by dividing the total number of correctly classified pixels by the total number of reference pixels (Lillesand and Kiefer, 1999). The kappa coefficient is calculated by using equation (Bishop et al., 1975) given as,

$\widehat{K}=\frac{N \sum_{i=1}^{n} X_{i i}-\sum_{i=1}^{n} x_{i+} X_{+i}}{N^{2}-\sum_{i=1}^{n} X_{i+} X_{+i}}$

Where,

$n=$ Number of rows and columns in a contingency matrix

$x_{i i}=$ The number of observations in $\mathrm{i}^{\text {th }}$ row and $\mathrm{i}^{\text {th }}$ column (major diagonal)

$x_{i+}=$ Total of observations in $\mathrm{i}^{\text {th }}$ row (right margin)

$x_{+i}=$ Total observations in $\mathrm{i}^{\text {th }}$ column (bottom margin)

$N=$ Total number of observations included in a matrix

\section{RESULTS AND DISCUSSION}

The class separability analysis is performed for the polarization combination used for the classification. Table 1 gives the ROI (class) separability values for different class pairs. Out of five major identified classes, urban land gives higher separability amongst all other class-pairs. Urban land gives high backscattering because of corner reflector and it leads to a unique signature. Vegetation and agricultural land give high backscattering in cross polarized bands due to volume scattering. These two classes show high separability as shown in Table 1. Fallow lands having relatively smooth texture leads to low backscattering. So, it gives low separability.

Supervised classification (SVM) method is applied on HH, HV and $\mathrm{HH}+\mathrm{HV}$ polarization combination. The classification results using SVM is presented in Figure 3 and the corresponding contingency matrix (or error matrix) is presented in Table 2. It compares the classification results with ground truth information and reports the producer's accuracy, user's accuracy, overall accuracy and kappa coefficient. The overall classification accuracy is estimated as $95.23 \%$ and kappa coefficient as 0.9350 . The urban land, agricultural land, water bodies and vegetation can be easily distinguished from each other. The fallow land cannot be easily identified because it is surrounded by other classes which lead to less optimum class boundaries.

\begin{tabular}{lll}
\hline & ROI (Class) Pair & $\begin{array}{l}\text { Transformed } \\
\text { Divergence }\end{array}$ \\
\hline Urban land & Agricultural land & 1.96310739 \\
Urban land & Vegetation & 1.99360141 \\
Urban land & Fallow land & 1.57469457 \\
Urban land & Water bodies & 1.99996567 \\
Agricultural land & Vegetation & 1.91258692 \\
Agricultural land & Fallow land & 1.99375053 \\
Agricultural land & Water bodies & 1.95129469 \\
Vegetation & Fallow land & 1.89109046 \\
Vegetation & Water bodies & 1.99999951 \\
Fallow land & Water bodies & 2.00000000 \\
\hline
\end{tabular}

Table 1. Class separability of various land use/cover class-pairs for RISAT-1 data using Transformed divergence procedure

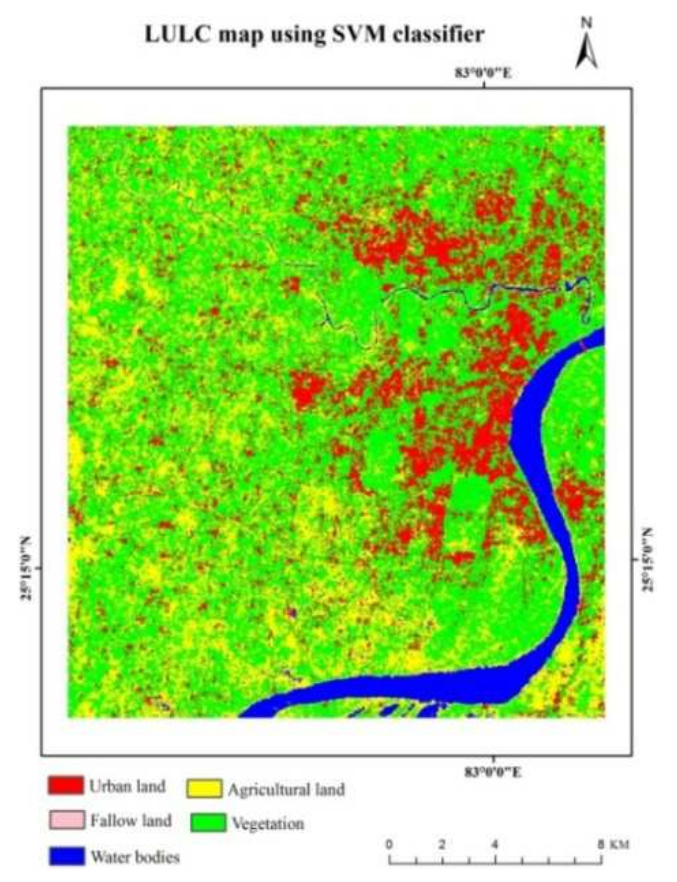

Figure 3. LULC map of RISAT-1 data using SVM classifier

\section{CONCLUSIONS}

The potential of SVM for land use land cover classification using dual polarimetric RISAT-1 data is evaluated in this paper. The class separability analysis is also performed to assess the quality of the training samples prior to image classification. Five classes are identified as urban land, agricultural land, fallow land, vegetation and water bodies. The result shows fairly good classification accuracy for each class. 


\begin{tabular}{|c|c|c|c|c|c|c|c|}
\hline LULC classes & Urban land & Water bodies & Agricultural land & Vegetation & Fallow land & Total & User's accuracy \\
\hline Urban land & 612 & 0 & 6 & 7 & 4 & 629 & 97.29 \\
\hline Water bodies & 1 & 748 & 3 & 0 & 0 & 752 & 99.46 \\
\hline Vegetation & 1 & 0 & 14 & 325 & 3 & 343 & 94.75 \\
\hline Fallow land & 4 & 0 & 1 & 3 & 32 & 40 & 80.00 \\
\hline Total & 654 & 759 & 386 & 345 & 39 & 2183 & \\
\hline
\end{tabular}

Table 2. Contingency table for SVM classifier

\section{ACKNOWLEDGMENT}

The authors wish to gratefully acknowledge Prof. Rajeev Sangal Director, Indian Institute of Technology (B.H.U.), Varanasi, for providing financial support to procure image processing software ENVI version 5.1 with SARscape module.

\section{REFERENCES}

Bishop, Y., Fienberg, S., Holland, P., 1975. Discrete multivariate analysis theory and practice. Massachusetts: MIT Press.

Congalton, R. G., Green, K., 1999. Assessing The Accuracy Of Remotely Sensed Data: Principles And Practices. CRC/Lewis Press, Boca Raton, pp. 74-79

Cortes, C., Vapnik, V. N., 1995. Support-vector networks. Machine Learning, 20, pp. 273-297.

Dixon, B., Candade, N., 2008. Multispectral land use classification using neural networks and support vector machines: one or the other, or both? International Journal of Remote Sensing, 29(4), pp. 1185-1206.

Foody, G. M., 2004. Supervised image classification by MLP and RBF neural networks with and without an exhaustively defined set of classes. International Journal of Remote Sensing, 25(15), pp. 3091-3104.

Foody, G. M., Mathur, A., 2004. A relative evaluation of multiclass image classification by support vector machines. IEEE Transactions on Geoscience and Remote Sensing, 42(6), pp. 1335-1343.

Fukuda, S., Hirosawa, H., 2001. Support vector machine classification of land cover: application to polarimetric sar data. Geoscience and Remote Sensing Symposium Proceedings, 187.

Hall, F. G., Townshend, J. R. G., Engman, E. T., 1995. Status of remote sensing algorithms for estimation of land surface state parameters. Remote Sensing of Environment 51, pp. 138-156.

Huang, C., Davis, L. S., Townshend, J. R. G., 2002. An assessment of support vector machines for land cover classification. International Journal of Remote Sensing 23(4), pp. 725-749.
Hsu, C.-W. and Lin, C.-J., 2002. A comparison of methods for multi-class support vector machines. IEEE Transactions on Neural Networks, 13(2), pp. 415-425.

Krishnapuram, B., Sichina, J., Carin, L., 2003. Physics-based detection of targets in sar imagery using support vector machines. IEEE Sensors Journal 3(2), pp. 147-157.

Lillesand, T. M., Kiefer, R. W., 1999. Remote Sensing And Image Interpretation. Wiley, New York.

Mercier, G., Lennon, M., 2000. Support vector machines for hyperspectral image classification with spectral based kernels. Geoscience and Remote Sensing Symposium Proceedings, 288.

Mountrakis, G., Im, J., Ogole, C., 2011. Support vector machines in remote sensing: a review. ISPRS Journal of Photogrammetry and Remote Sensing, 66(3), pp. 247-259.

Perkins, S. J., Harvey, N. R., Brumby, S. P., Lacker, K., 2001. Support Vector Machines for Broad Area Feature Extraction in Remotely Sensed Images. Proc. SPIE 4381 Orlando, FL, April 16.

Pierce, L. E., Bergen, K. M., Dobson, M. C., Ulaby, F. T., 1998. Multitemporal landcover classification using SIR-C/X-SAR imagery. Remote Sensing of Environment, 64, pp. 20-33.

Roberts, D. A., Keller, M., Soares, J. V., 2003. Studies of landcover, land-use, and biophysical properties of vegetation in the large scale biosphere atmosphere experiment in Amazonia. Remote Sensing of Environment, 87, pp. 377-388.

Saatchi, S. S., Soares, J. V., Alves, D. S., 1997. Mapping deforestation and land use in amazon rainforest by using SIR-C imagery. Remote Sensing of Environment, 59, 191-202.

Thenkabail, P. S., Schull, M., Turral, H., 2005. Ganges and Indus river basin land use/ land cover (LULC) and irrigated area mapping using continuous streams of MODIS data. Remote Sensing of Environment, 95, pp. 317-341.

Vapnik, V. N., 1995. The Nature Of Statistical Learning Theory. Springer-Verlag, New York, NY

Vapnik, V. N., 1998. Statistical Learning Theory. Willey, New York, 768p.

Vapnik, V. N., 1999. An overview of statistical learning theory. IEEE Transactions on Neural Networks, 10(5), pp. 988-999. 
Waske, B., Benediktsson, J. A., 2007. Fusion of support vector machines for classification of multisensor data. IEEE Transactions on Geoscience and Remote Sensing, 45(12), pp. 3858-3866.

Waske, B., Braun, M., 2009. Classifier ensembles for land cover mapping using multitemporal sar imagery. ISPRS journal of Photogrammetry and Remote Sensing, 64(5), pp. 450-457. 